\title{
BOUNDARY VALUE PROBLEMS FOR PSEUDOANALYTIC FUNCTIONS
}

\author{
BY WALTER KOPPELMAN ${ }^{1}$ \\ Communicated by Lipman Bers, March 8, 1961
}

The object of this note is the discussion of certain linear boundary value problems associated with the theory of linear elliptic systems of partial differential equations in two independent variables. Such problems were treated for multiply connected plane domains by I. N. Vekua [10], who successfully applied a technique utilizing singular integral equations. The results suggested the possibility of studying these problems for domains which are not schlicht. Unfortunately, the methods developed by Vekua did not lend themselves to generalization without scrutiny (see [8]). It appeared best, therefore, to restudy the question from an entirely different viewpoint. This involved an independent treatment of boundary value problems for analytic functions $[3 ; 4 ; 7 ; 9]$. Below, we shall indicate how results for such problems in the theory of analytic functions may be transferred to results for corresponding problems in the theory of pseudoanalytic functions. Proofs and further theorems will appear elsewhere.

1. Formulation of the Riemann-Hilbert problem. Letting $D$ denote a given Riemann surface of finite genus, with a boundary $\dot{D}$ consisting of a finite number of Liapounov curves, i.e., curves with Hölder continuously turning tangents, and interior $D_{0}$, we shall now pose a boundary value problem which we denote by $\operatorname{RH}(a, b, \gamma, \Lambda)$. Here $\Lambda$ and $\gamma$ are to be given functions on $\dot{D}$, with $|\Lambda|=1$ and $\gamma$ real. Furthermore, $\Lambda$ is to be Hölder continuously differentiable, while $\gamma$ is assumed to be Hölder continuous. $a$ and $b$ are given coefficients of a conjugate differential on $D_{0}$, i.e., $a d \bar{z}$ and $b d \bar{z}$ are invariant under conformal transformations. We assume here that $a$ and $b$ are bounded and measurable in the sense that if $g$ is the coefficient of a nonvanishing, continuous differential on $D$, i.e., $g d z$ is invariant under conformal transformations, then $a / \bar{g}, b / \bar{g}$ are bounded, measurable functions on $D_{0}$. The problem $\operatorname{RH}(a, b, \gamma, \Lambda)$ will consist of finding functions $w$ continuous on $D$, such that

$$
w_{\bar{z}}=a w+b \bar{w}
$$

in $D_{0}$, and

1 The research presented here was supported by the National Science Foundation under grant No. NSF-G14445. 


$$
\operatorname{Re}(\Lambda w)=\gamma
$$

on $\dot{D}$. Because of the lack of a hypothesis concerning the smoothness of the coefficients $a$ and $b$, the relation (1.1) must, as usual, be interpreted in a weak sense.

Together with the problem $\mathrm{RH}(a, b, \gamma, \Lambda)$, we shall also consider the homogeneous adjoint boundary value problem $\mathrm{RH}^{*}(a, b, 0, \Lambda)$ for continuous coefficients $v$ of differentials, which are to satisfy the relations

$$
v_{\bar{z}}=-a v-\bar{b} \bar{v}
$$

in $D_{0}$, and

$$
\operatorname{Re}\left(\bar{\Lambda} v \frac{d z}{d s}\right)=0
$$

on $\dot{D}$.

2. The operators $T$ and $T^{*}$. Let $\left\{W_{j}\right\}, j=1, \cdots, A$ be a complete set of linearly independent solutions of $\operatorname{RH}(0,0,0, \Lambda)$; let $\left\{V_{l}\right\}$, $l=1, \cdots, B$ be a complete set of linearly independent solutions of $\mathrm{RH}^{*}(0,0,0, \Lambda)$. Define the functions

$$
\begin{array}{ll}
h_{j}=i \Lambda W_{j}, & j=1, \cdots, A ; \\
k_{l}=i \bar{\Lambda} V_{l} \frac{d z}{d s}, & l=1, \cdots, B,
\end{array}
$$

on $\dot{D}$. Suppose that the functions $\hat{k}_{l}, l=1, \cdots, B ; \hat{h}_{j}, j=1, \cdots, A$, are a set of Hölder continuously differentiable, real-valued functions on $\dot{D}$ with the property that the matrices

$$
\left(\int_{\dot{D}} \hat{k}_{l} k_{m} d s\right), \quad l, m=1, \cdots, B
$$

and

$$
\left(\int_{\dot{D}} \hat{h}_{h} h_{n} d s\right), \quad j, n=1, \cdots, A
$$

are of rank $B$ and $A$, respectively. We then define linear operators $T$ and $T^{*}$ through the requirements

$$
\begin{aligned}
(T w)_{\bar{z}} & =a w+b \bar{w} \quad \text { in } D_{0}, \\
\operatorname{Re} \Lambda T w & =\sum_{l=1}^{B} \kappa_{l} \hat{k}_{l} \quad \text { on } D,
\end{aligned}
$$




$$
\begin{aligned}
& \int_{\dot{D}} \hat{h}_{j}(\operatorname{Im} \Lambda T w) d s=0, \quad j=1, \cdots, A, \\
& \left(T^{*} v\right)_{\bar{z}}=-a v-\bar{b} \bar{v} \quad \text { in } D_{0},
\end{aligned}
$$$$
\operatorname{Re} \bar{\Lambda} \frac{d z}{d s} T^{*} v=\sum_{j=1}^{A} \lambda_{j} \hat{h}_{j} \text { on } D,
$$

$$
\int_{\dot{D}} \hat{k}_{l}\left(\operatorname{Im} \bar{\Lambda} \frac{d z}{d s} T^{*} v\right) d s=0, \quad l=1, \cdots, B
$$

These operators are at first defined for $w$ and $v$ which are continuously differentiable on $D$. The relations (2.4) and (2.7) are to be understood to hold in a generalized sense. The quantities $\kappa_{l}$ and $\lambda_{j}$ are suitable real constants, uniquely determined by $w$ and $v$ in such a way, that $T w$ and $T^{*} v$ may always be defined. The operators $T$ and $T^{*}$ are "adjoints" with respect to the "scalar product"

$$
[w, v]=\operatorname{Im} 2 i \iint_{D}(a v+b \bar{v}) w d x d y .
$$

3. A coercive inequality. In his work on elliptic equations, Martin Schechter showed that boundary conditions of the type considered here, for first order elliptic equations, lead to coercive inequalities [6]. In order to state this result for the special problems here, we introduce the norms

$$
\begin{aligned}
& \|w\|_{0}^{2}=\iint_{D}|w|^{2}|g|^{2} d x d y, \\
& \|w\|_{1}^{2}=\|w\|_{0}^{2}+\iint_{D}\left\{\left|w_{x}\right|^{2}+\left|w_{y}\right|^{2}\right\} d x d y
\end{aligned}
$$

for functions $w$ on $D$,

$$
\langle\gamma\rangle_{1}^{2}=\int_{\dot{D}}\left\{|\gamma|^{2}+\left|\frac{d \gamma}{d s}\right|^{2}\right\} d s,
$$

for functions $\gamma$ on $\dot{D}$, and

$$
\|v\|_{0}^{2}=\iint_{\dot{D}}|v|^{2} d x d y
$$

for differentials $v d z$, or conjugate differentials $v d \bar{z}$ on $D$. Here $g$ is to be an arbitrary but, from now on, fixed coefficient of a nonvanishing continuous differential on $D$. We then have 
Lemma 3.1. There exists a constant $K$ which depends only on $\Lambda$ and $D$, such that for all functions $w$, continuously differentiable on $D$,

$$
\|w\|_{1}^{2} \leqq K\left(\left\|w_{\bar{z}}\right\|_{0}^{2}+\langle\operatorname{Re} \Lambda w\rangle_{1}^{2}+\|w\|_{0}^{2}\right) .
$$

Next, we introduce the Hilbert space ${ }^{0} H_{0}$ of square summable functions $w$ with norms $\|w\|_{0}$ and also the Hilbert space ${ }^{1} H_{0}$ of square summable differentials $v d z$ with norms $\|v\|_{0}$. With the aid of Lemma 3.1 and Rellich's lemma [2], one can now prove

LEMмA 3.2. The operator $T\left(T^{*}\right)$ may be extended to the entire Hilbert space ${ }^{0} \mathrm{H}_{0}\left({ }^{1} \mathrm{H}_{0}\right)$ as a compact linear operator.

We now remark that, although $T^{*}$ appears to be the adjoint of $T$ with respect to a representation of the bounded linear functionals on ${ }^{\circ} H_{0}$ of the form (2.10), extreme care must be exercised, since this representation (2.10) is not faithful. However, the theory of pseudoanalytic functions itself, together with the particular definitions of the operators $T$ and $T^{*}$, lead to the conclusion that the usual Fredholm theorems governing the nature of the null spaces and ranges of the operators $1-T$ and $1-T^{*}$ hold.

4. The main theorems. In order to prove the main theorems, we use a theorem on the regularity of the solutions of the Fredholm equations, which we state as

LеммA 4.1. If $W$ is analytic in $D_{0}$ and Hölder continuous on $D$, and if $w$ is a function in ${ }^{\circ} \mathrm{H}_{0}$ satisfying

$$
w-T w=W,
$$

then $w$ is also Hölder continuous ${ }^{2}$ on $D$ and satisfies (1.1) in $D_{0}$.

An analogous statement holds for the operator $T^{*}$.

Our first basic result is contained in

TheOREM 4.2. The problems $R H(a, b, 0, \Lambda)$ and $R H^{*}(a, b, 0, \Lambda)$ possess only finitely many linearly independent solutions. If $A$ and $B$ denote the number of linearly independent solutions of the problems $R H(0,0,0, \Lambda), R H^{*}(0,0,0, \Lambda)$ respectively, while $A^{\prime}$ and $B^{\prime}$ denote the number of linearly independent solutions of $R H(a, b, 0, \Lambda)$ and $R H^{*}(a, b, 0, \Lambda)$, then

$$
A-B=A^{\prime}-B^{\prime}
$$

The second result concerns the solvability of problems with inhomogeneous boundary data.

\footnotetext{
2 Possibly only after correction on a set of measure zero.
} 
TheOREM 4.3. The problem $R H(a, b, \gamma, \Lambda)$ possesses a solution if, and only if,

$$
\int_{\dot{D}} \bar{\Lambda} \gamma v d z=0
$$

for every solution $v$ of $R H^{*}(a, b, 0, \Lambda)$.

5. The Hilbert problem. ${ }^{3}$ It is important to observe that the techniques which we have applied to the problems $\mathrm{RH}(a, b, \gamma, \Lambda)$ may also be used for an entirely different set of problems $\mathrm{H}(a, b, g, G)$, which we now formulate. In this case, we work with a closed Riemann surface $R$ and a system $L$ of closed, disjoint and oriented Liapounov curves. Once these curves are given a definite orientation, we may associate a left or $(+)$ edge and a right or $(-)$ edge with $L$. Here $G$ and $g$ are given complex-valued functions on $L$, with $G \neq 0$. We furthermore assume that $G$ is Hölder continuously differentiable, while $g$ is Hölder continuous. The problem $\mathrm{H}(a, b, g, G)$ now consists of finding functions $w$, piecewise continuous on $R$ and continuous on $R-L$, which satisfy (1.1) on $R-L$ and the jump condition

$$
w^{+}=G w^{-}+g
$$

across $L$. Together with $\mathrm{H}(a, b, g, G)$, we again consider a homogeneous adjoint problem $\mathrm{H}^{*}(a, b, 0, G)$ for piecewise continuous differentials $v d z$ satisfying (1.3) on $R-L$, and the jump condition

$$
v^{+}=G^{-1} v^{-}
$$

across $L$. One may obtain an analogue of Theorem 4.2. The result corresponding to Theorem 4.3 is now stated as

Theorem 5.1. The problem $H(a, b, g, G)$ possesses a solution if, and only if

$$
\operatorname{Im} \int_{\dot{D}} g v^{+} d z=0
$$

for every solution $v$ of $H^{*}(a, b, 0, G)$.

As an interesting consequence of the theorems in this section and information concerning similar problems for the Cauchy-Riemann equations [4], we obtain

Theorem 5.2 (RIEMANN-Roch Theorem For PSEudoanalytic FUNCTIONS). Let $R$ be a closed Riemann surface of genus $h$ and $d$ a divi-

\footnotetext{
Some previous work concerning the Hilbert problem for differential equations on spheres can be found in [5].
} 
sion on $R$, of order $n$. Let $A$ be the number of linearly independent solutions of (1.1) which are multiples of $d^{-1}$ on $R$ and let $B$ be the number of linearly independent solutions of (1.3) which are multiples of $d$ on $R$. Then

$$
A-B=2(n-h+1) \text {. }
$$

This generalizes a result of Bers [1] who stated this Riemann-Roch theorem under the additional assumption that equation (1.1) possesses two linearly independent solutions which are regular everywhere on $R$.

6. Concluding remarks. The essential feature of this paper is the utilization of variational techniques to solve the boundary value problems which we have stated, in the large. One disadvantage of this method, however, lies in the necessity of requiring excessive smoothness conditions on the boundary data, in this case, the functions $\Lambda$ and $G$. A comparison of our results with those of Vekua [10], in the case of the Riemann-Hilbert problem for plane domains, reveals this fact quite clearly. It is to be hoped that further work will remove such restrictions.

\section{BIBLIOGRAPHY}

1. L. Bers, An outline of the theory of pseudoanalytic functions, Bull. Amer. Math. Soc. vol. 62 (1956) pp. 291-329.

2. R. Courant and D. Hilbert, Methoden der mathematischen Physik, vol. 2, Berlin, J. Springer, 1937.

3. W. Koppelman, The Riemann-Hilbert problem for finite Riemann surfaces, Comm. Pure Appl. Math. vol. 12 (1959) pp. 13-35.

4. - - Singular integral equations, boundary value problems and the RiemannRoch theorem, J. Math. Mech. vol. 10 (1961) pp. 247-277.

5. L. G. Mihaylov, $A$ boundary problem of the type of Riemann for systems of first order differential equations of elliptic type, Dokl. Akad. Nauk SSSR (N. S.) vol. 112 (1957) pp. 13-15.

6. M. Schechter, On estimating partial differential operators. III, Abstract 538-19, Notices Amer. Math. Soc. vol. 5 (1958) p. 28.

7. Yu. L. Rodin, Conditions for the solvability of the Riemann and Hilbert boundary value problems on Riemann surfaces, Dokl. Akad. Nauk SSSR (N. S.) vol. 129 (1959) pp. 1234-1237.

8. - The characteristic functions of certain integral equations, Dokl. Akad. Nauk SSSR (N. S.) vol. 130 (1960) pp. 23-25.

9. - On the Riemann problem on closed Riemann surfaces, Dokl. Akad. Nauk SSSR (N. S.) vol. 132 (1960) pp. 1038-1040.

10. I. N. Vekua, Systems of partial differential equations of first order of elliptic type and boundary value problems with applications to the theory of shells, Mat. Sb. (N.S.) vol. 31 (1952) pp. 217-314.

University of Pennsylvania 